\title{
Funções de agregação baseadas em integral de Choquet aplicadas em redimensionalização de imagens
}

\author{
Jéssica C. S. Bueno ${ }^{1}$, Camila A. Dias ${ }^{2}$, Graçaliz P. Dimuro ${ }^{1,2}$, Eduardo N. Borges ${ }^{2}$, Silvia S. \\ C. Botelho ${ }^{1,2}$, Viviane L. D. de Mattos ${ }^{1}$ and Humberto Bustince ${ }^{3}$ \\ ${ }^{1}$ Programa de Pós-Graduação em Modelagem Computacional, FURG and ${ }^{2}$ Programa de Pós-Graduação em Computação, FURG and \\ ${ }^{3}$ Dpt. de Automatica y Computacion, UPNA \\ *jessica_bsaldivia@hotmail.com; cmdias@outlook.com.br; \{enborges, gracaliz, silviacb.botelho, viviane.leite.mattos\}@gmail.com; bustince@unavarra.es
}

Recebido: 05/02/2019. Revisado: 08/02/2019. Aceito: 01/03/2019.

\begin{abstract}
Resumo
O crescente aumento do volume de dados, juntamente com a alta complexidade destes, tem gerado a necessidade de se desenvolver técnicas de extração de conhecimento cada vez mais eficientes, tanto em custo computacional quanto em precisão. A maioria dos problemas que são tratados por esses técnicas tem informações complexas a serem identificadas. Para isso são utilizados métodos de aprendizado de máquina, onde esses métodos usam uma variedade de funções dentro das diferentes etapas que são empregadas em suas arquiteturas. Uma dessas consiste no uso de funções de agregação para redimensionar imagens. Neste contexto, apresenta-se um estudo de funções de agregação baseadas na integral de Choquet, onde a principal característica da integral do Choquet, em comparação com outras funções de agregação, reside no fato de que ela considera, por meio da medida fuzzy, a interação entre os elementos a serem agregados. Logo, apresenta-se um estudo avaliativo do desempenho das funções integral de Choquet clássica, integral de Choquet baseada em Cópula em relação as funções máximo e média, procurando resultados que podem ser melhores do que as funções de agregação usualmente aplicadas. Os resultados de tais comparações são promissores, quando avaliados através de medidas de qualidade de imagem.
\end{abstract}

Palavras-Chave: Funções de Agregação; integral de Choquet; processamento de imagens

\begin{abstract}
The increasing data volume, coupled with the high complexity of these data, has generated the need to develop increasingly efficient knowledge extraction techniques, both in computational cost and precision. Most of the problems that are addressed by these techniques have complex information to be identified. For this, machine learning methods are used, where these methods use a variety of functions inside the different steps that are employed in their architectures. One of these consists in the use of aggregation functions to resize images. In this context, a study of aggregation functions based on the Choquet integral is presented, where the main feature of Choquet integral, in comparison with other aggregation functions, resides in the fact that it considers, through the fuzzy measure, the interaction between the elements to be aggregated. Thus, an evaluation study of the performance of the standard Choquet integral functions is presented (Choquet integral based on Copula in relation to the maximum and average functions) looking for results that may be better than the usual applied aggregation functions. The results of such comparisons are promising when evaluated through measures of image quality.
\end{abstract}

Key words: Aggregation functions; Choquet integral; image processing.

\section{Introdução}

O crescente aumento do volume de dados, juntamente com a alta complexidade destes, tem gerado a necessidade de se desenvolver técnicas de extração de conhecimento cada vez mais eficientes, tanto em custo computacional quanto em precisão. A classificação é uma das técnicas mais utilizadas no contexto de extração de conhecimento de dados sendo aplicada em diversos campos, como saúde, criminal, entre outros.

A classificação de imagens é um problema muito comum na área de Visão Computacional, onde os principais problemas enfrentados são, por exemplo, identificar padrões em imagens, 
distinguir seres vivos e objetos, rotular imagens coletadas, entre outros. A maioria desses problemas tem informações complexas a serem identificadas.

Esses métodos usam uma variedade de funções dentro das diferentes etapas que são empregadas em suas arquiteturas. Uma dessas consiste no uso de funções de agregação ${ }^{1}$, como o máximo ou média aritmética (Scherer et al.; 2010). Para aperfeiçoar a agregação de informações significativas sem degradar seu poder discriminativo no processamento de imagens, propõe-se a substituição da função máximo e média, usuais na literatura, pela integral de Choquet (Choquet; 1999).

A integral de Choquet, neste contexto, tem como domínio os valores dos pixels de uma imagem de entrada, que variam de 0 a 255. Para realizar a medição da qualidade da imagem de saída foram utilizadas medidas de qualidade de imagem usuais na literatura. Além disso, para facilitar a análise dos resultados, as imagens de entrada estão em escala de cinza.

Dessa forma, o objetivo deste trabalho é apresentar um estudo da aplicação de funções de agregação baseadas na integral de Choquet para reduzir o tamanho de uma imagem. Esse estudo está sendo realizado aplicando as funções de agregação (integral de Choquet clássica, bem como a integral de Choquet baseada em Cópula ) em um grupo de imagens, obtendo os resultados de cada imagem individualmente e comparando os resultados com os das funções máximo e média aritmética.

As comparações dos resultados das imagens de entrada com as de saída são feitas através de funções de medida de qualidade de imagem. Pretende-se avaliar o desempenho da integral de Choquet clássica, bem como a integral de Choquet baseada em Cópula em relação as funções máximo e média. Ou seja, deseja-se avaliar se as funções baseadas em integral de Choquet podem superar ou apresentar possibilidades de serem melhores do que as funções máximo e média, usuais na literatura.

Este artigo está organizado da seguinte maneira. Na Seção 2, apresentam-se alguns conceitos preliminares tais como a definição de função de agregação, além das definições da integral de Choquet e integral de Choquet baseada em Cópula. Na Seção 3, é exposta a formulação do problema, onde é detalhado o processo de redimensionalização de imagens por funções de agregação. As análises dos resultados experimentais são apresentadas na Seção 4 Por fim, na Seção 5, apresentam-se as principais considerações do trabalho.

\section{Conceitos Preliminares}

Agregação é um processo de combinar diferentes valores numéricos retornando um único valor. A regra que realiza essa tarefa é denominada função de agregação (Grabisch et al.; 2009). Os valores de entrada de uma função de agregação podem ser, por exemplo, graus de pertinência de conjuntos fuzzy, peso de critérios, notas, graus de preferência, etc. Sendo assim, sua aplicação ocorre em diversas áreas das ciências exatas como estatística, ciência da computação, matemática, economia entre outras.

Definição 1 Uma função $\mathcal{A}:[0,1]^{n} \rightarrow[0,1]$ de aridade $n e ́$ dita de agregação se satisfaz as seguintes condições:

$$
\begin{aligned}
& \text { (A1) Condições de contorno: } \mathcal{A}(\overbrace{0,0, \ldots, 0}^{n-v e z e s})=0 e \\
& \mathcal{A}(\overbrace{1,1, \ldots, 1}^{n-v e z e s})=1 .
\end{aligned}
$$$$
\text { (A2) Monotonicidade: } \mathcal{A}\left(x_{1}, x_{2}, \ldots, x_{n}\right) \leq \mathcal{A}\left(y_{1}, y_{2}, \ldots, y_{n}\right)
$$$$
\text { sempre que } x_{i} \leq y_{i} \text { para todo } i \in\{1, \ldots, n\} \text {, ou seja, } \mathcal{A} \text { é não- }
$$

\footnotetext{
${ }^{1}$ no contexto de processamento de imagens utiliza-se o termo pooling em vez de agregação, porém neste trabalho será adotado o termo agregação devido às funções de agregação
}

\section{decrescente em cada argumento.}

A função de agregação definida no domínio $[0,1]^{n}$ e contra-domínio $[0,1]$ visa o contexto fuzzy. Poderia ser considerado um intervalo fechado da reta real diferente desse. As funções de agregação mais comuns na literatura são as funções de máximo, que pode ser definida como $f\left(x_{1}, x_{2}, \ldots, x_{n}\right)=\max \left\{x_{1}, x_{2}, \ldots, x_{n}\right\}$, e a função mínimo definida por $f\left(x_{1}, x_{2}, \ldots, x_{n}\right)=\min \left\{x_{1}, x_{2}, \ldots, x_{n}\right\}$. Além, é claro, da tão conhecida função média aritmética.

Neste trabalho, é apresentado uma função de agregação alternativa as clássicas que é a integral de Choquet (Beliakov et al.; 2016). Esta é uma extensão da integral de Lebesgue, sendo definida com base em uma medida fuzzy. A medida fuzzy possui várias interpretações dependendo do contexto do problema a ser trabalhado.

No contexto das funções de agregação, a medida fuzzy representa o grau de relação entre os elementos a serem agregados (BELIAKOV, SOLA, \& SÁNCHEZ, 2016). Desta forma, o grande uso da integral do Choquet ocorre devido ao seu modelo para considerar a importância de cada atributo a ser agregado, bem como suas interações.

Definição 2 Seja $N=\{1, \ldots, n\}$ e $2^{N}$ o conjunto das partes de $N$. A função $\mathfrak{m}: 2^{N} \rightarrow[0,1]$ é uma medida fuzzy se, para todo conjunto $A, B \subseteq N$, as seguintes condições são satisfeitas: (m1) Condições de Contorno: $\mathfrak{m}(\emptyset)=0$ e $\mathfrak{m}(N)=1$. (m2) Monotonicidade: $\mathfrak{m}(A) \leq \mathfrak{m}(B)$ sempre que $A \subseteq B$.

Definição 3 Seja $\mathfrak{m}: 2^{N} \rightarrow[0,1]$ uma medida fuzzy. A integral de Choquet discreta de $\overrightarrow{\boldsymbol{x}}=\left(\mathrm{x}_{1}, \mathrm{x}_{2}, \ldots, \mathrm{x}_{n}\right) \in[0,1]^{n}$ com relação a medida fuzzy $\mathfrak{m}$ é uma função $\mathfrak{C}_{\mathfrak{m}}:[0,1]^{n} \rightarrow[0,1]$, definida por

$$
\mathfrak{C}_{\mathfrak{m}}(\vec{x})=\sum_{i=1}^{n}\left(x_{(i)}-x_{(i-1)}\right) \cdot \mathfrak{m}\left(A_{(i)}\right)
$$

onde $\vec{x}_{\nearrow}=\left(x_{(1)}, \ldots, x_{(n)}\right)$ é uma permutação não-decrescente de $\vec{x}$, isto é, $0 \leq \chi_{(1)} \leq \ldots \leq x_{(n)}$, por convenção $\chi_{(0)}=0$, $e$ $A_{(i)}=\{(i), \ldots,(n)\}$ é um subconjunto dos índices das $n-i+1$ maiores componentes de $\vec{x}$.

A integral de Choquet Eq.(1) assim definida satisfaz as condições da Def. 1, logo é uma função de agregação. Além disso, a integral de Choquet pode ser escrita na forma expandida como:

$$
\mathfrak{C}_{\mathfrak{m}}(\vec{x})=\sum_{i=1}^{n}\left(x_{(i)} \cdot \mathfrak{m}\left(A_{(i)}\right)-x_{(i-1)} \cdot \mathfrak{m}\left(A_{(i)}\right)\right)
$$

Ressalta-se que a principal característica da integral do Choquet, em comparação com outras funções de agregação, reside no fato de que ela considera, por meio da medida fuzzy, a interação entre os elementos a serem agregados. Por exemplo, o máximo não considera a relação entre os elementos a serem agregados, descartando informações importantes entre esses elementos.

No contexto de processamento de imagem, os valores a serem agregados são os pixels que aparecem em uma janela. Assim, neste sentido, entende-se que quanto mais informações das relações entre os pixels a serem agregados, melhor será a imagem de saída.

A integral de Choquet pode ser generalizada por Cópulas (Alsina et al.; 2006), gerando uma família de funções de agregação denominadas CC-integrais (Lucca et al.; 2017). Essa generalização é construída trocando-se o operador do produto da integral de Choquet na forma expandida Eq. (2) por uma Cópula. 
Definição 4 Seja $\mathfrak{m}: 2^{N} \rightarrow[0,1]$ uma medida fuzzy $e$ C : $[0,1]^{2} \rightarrow[0,1]$ uma Cópula. A integral de Choquet discreta baseada em Cópula com relação a medida fuzzy $\mathfrak{m}$ é uma função $\mathfrak{C}_{\mathfrak{m}}^{C}:[0,1]^{n} \rightarrow[0,1]$, definida por

$$
\mathfrak{C}_{\mathfrak{m}}^{C}(\vec{x})=\sum_{i=1}^{n} C\left(x_{(i)}, \mathfrak{m}\left(A_{(i)}\right)\right)-C\left(x_{(i-1)}, \mathfrak{m}\left(A_{(i)}\right)\right),
$$

onde $\vec{x}_{\nearrow}=\left(x_{(1)}, \ldots, x_{(n)}\right)$ é uma permutação não-decrescente de $\vec{x}$, isto é, $0 \leq \chi_{(1)} \leq \ldots \leq \chi_{(n)}$, por convenção $\chi_{(0)}=0$, $e$ $A_{(i)}=\{(i), \ldots,(n)\}$ é um subconjunto dos índices das $n-i+1$ maiores componentes de $\vec{x}$. Observa-se que a Eq. (3) satisfaz as condições da Def. 1, logo $\mathfrak{C}_{\mathfrak{m}}^{C}$ também é uma função de agregação.

Aplicando-se na Eq. (3) a Cópula T-norma do mínimo (Klement et al.; 2000), obtém-se a seguinte função:

$\mathfrak{C}_{\mathfrak{m}}^{T_{M}}(\vec{x})=\sum_{i=1}^{n}\left(\min \left\{x_{(i)}, \mathfrak{m}\left(A_{(i)}\right)\right\}-\min \left\{x_{(i-1)}, \mathfrak{m}\left(A_{(i)}\right)\right\}\right)$.

A medida fuzzy adotada neste trabalho é a medida da potência, definida por

$$
\mathfrak{m}_{P}(A)=\left(\frac{|A|}{n}\right)^{q}, \text { onde } q>0
$$

O valor de $q$, pode ser definido pelo especialista ou aprendido através de algum algoritmo computacional que é capaz de determinar o melhor valor para ele conforme o problema que esta sendo tratado.

\section{Formulação do Problema e Metodologia}

Para o estudo dos agregadores em redução dimensional de imagens foram consideradas as funções máximo e média usuais na literatura. Por outro lado, a escolha das funções de agregação baseadas na integral de Choquet foi feita com base no desempenho que obtiveram em Sistemas de Classificação Baseados em Regras Fuzzy publicado em trabalhos recentes. Tais funções são:

$$
\mathfrak{C}_{\mathfrak{m}}-\text { Eq. (1) e } \mathfrak{C}_{\mathfrak{m}}^{T_{M}}-\text { Eq. (4) }
$$

Nas funções baseadas na integral de Choquet, citadas acima, considerou-se a medida fuzzy da potência Eq. (5).

Objetivando estudar os agregadores, foram escolhidas, aleatoriamente, 12 imagens (Figura 1) do banco de dados IIIT $5 K$-Word dataset e, posteriormente, convertidas para escala de cinza através da função rgb2gray do software MatLab.

Para cada uma dessas 12 imagens, foram realizados experimentos considerando janelas de tamanhos $2 \times 2,3 \times 3$ e $4 \times 4$, e stride 2 e 3. Além disso, variou-se o parâmetro $q$ da medida fuzzy da potência Eq. (5) considerando-se os valores $q=0.1, q=0.3, q=0.5$ e $q=0.7$.

[Etapa 1] Redução Dimensional das Imagens:

Cada imagem de entrada gerou 144 imagens reduzidas, uma para cada combinação distinta das configurações de janela, stride, expoente da medida fuzzy da potência e agregador. Portanto, considerando as 12 imagens de entrada, foram geradas 288 imagens reduzidas para cada uma das quatro funções de agregação consideradas.

[Etapa 2] Restauração da Dimensão:
Na sequência emprega-se o resize $e^{2}$, utilizando a técnica Nearest Neighbor, a fim de retornar à mesma dimensão da imagem de entrada. O resize é utilizado após agregação com o propósito de comparação da imagem de entrada com a imagem que foi reduzida pela agregação, visto que só é possível aplicar medidas de qualidade de imagens uma vez que a imagem de entrada tenha a mesma dimensão da imagem de saída.

[Etapa 3] Análise por Medidas de Qualidade

A fim de realizar comparações das imagens de entrada com as imagens de saída, após o redimensionamento, foram aplicadas sete medidas de qualidade de imagem (Eskicioglu and Fisher; 1995):

- Average Difference (AD, resultado deve ser próximo ou igual a zero),

- Structural Content (SC $\downarrow$ ),

- Normalized Cross-Correlation (NK $\uparrow)$,

- Maximum Difference (MD $\downarrow$ ),

- Normalized Absolute Error (NAE $\downarrow$ ),

- Mean Squared Error (MSE $\downarrow$ ) e

- Peak Signal to Noise Ratio (PSNR $\uparrow$ ).

Essas medidas tem por finalidade mensurar o quanto a imagem de saída se aproxima ou a semelha da imagem de entrada. A flecha $\uparrow$ denota que quanto maior melhor a qualidade, ou seja, mais similares são as imagens. Por outro lado, $\downarrow$ indica que quanto menor melhor a aproximação das imagens. Para cada medida de qualidade de imagem obtém-se uma tabela com os resultados da comparação das imagens originais feitas com as respectivas imagens agregadas.

Definição 5 (Eskicioglu and Fisher; 1995) Seja uma imagem de dimensão $I \times J$, onde I é o número de linhas e J o número de colunas. Além disso, $P(i, j)$ representa a imagem original e $\widehat{P}(i, j)$ representa a imagem modificada. Ou seja, $P(i, j)$ e $\widehat{P}(i, j)$ representam o nivel de cinza da imagem original e modificada, respetivamente, no ponto $(i, j)$. Assim, pode-se definir as seguintes medidas de qualidade de imagem:

Average Difference (AD):

$$
A D=\frac{1}{M N} \sum_{i=1}^{I} \sum_{j=1}^{J}(P(i, j)-\widehat{P}(i, j))
$$

Maximum Difference $(\mathrm{MD} \downarrow)$ :

$M D=\operatorname{Max}|P(i, j)-\widehat{P}(i, j)|$, onde $i$ varia de 1 até $I$ e $j$ de 1 até $J$.

Mean Squared Error (MSE $\downarrow$ ):

$$
M S E=\frac{1}{M N} \sum_{i=1}^{I} \sum_{j=1}^{J}(P(i, j)-\widehat{P}(i, j))^{2}
$$

Normalized Absolute Error (NAE $\downarrow$ ):

$$
N A E=\frac{\sum_{i=1}^{I} \sum_{j=1}^{J} \mid O((P(i, j))-O(\widehat{P}(i, j)) \mid}{\sum_{i=1}^{I} \sum_{j=1}^{J}|O(P(i, j))|}
$$

Normalized Cross-Correlation ( $\mathrm{NK} \uparrow$ ):

$$
N K=\frac{\sum_{i=1}^{I} \sum_{j=1}^{J}(P(i, j) \times \widehat{P}(i, j))}{\sum_{i=1}^{I} \sum_{j=1}^{J}(P(i, j))^{2}}
$$




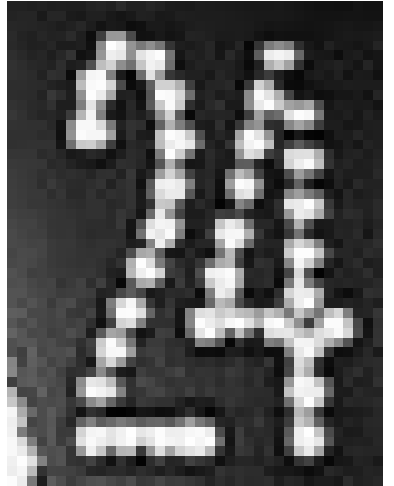

Imagem52_1

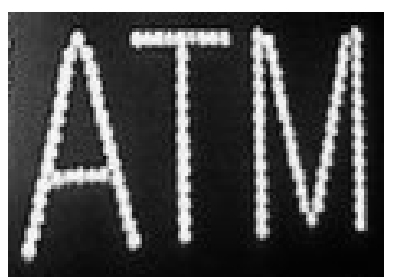

Imagem52_3

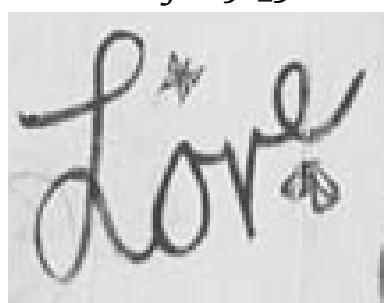

Imagem127_2

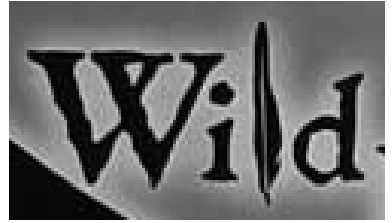

Imagem138_4

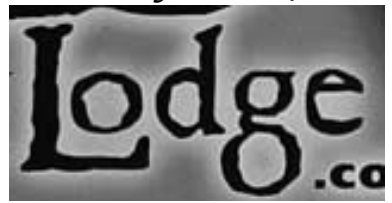

Imagem138_6

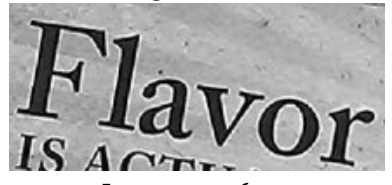

Imagem161_1

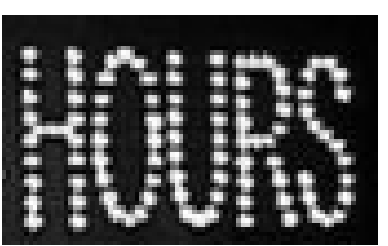

Imagem52_2

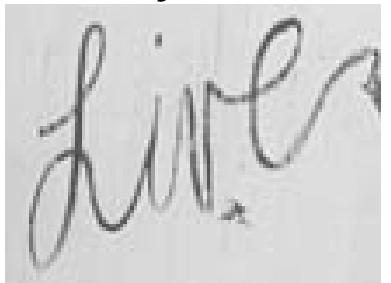

Imagem127_1

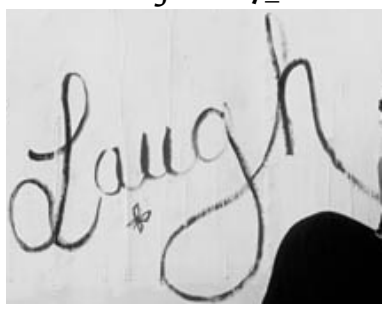

Imagem127_3

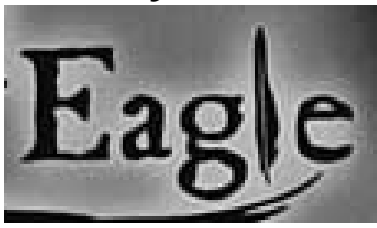

Imagem138_5

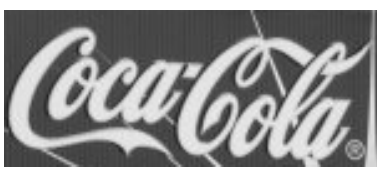

Imagem159_1

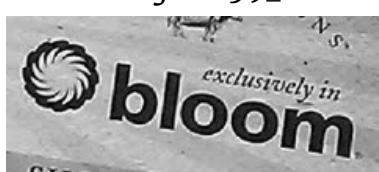

Imagem161_2

Figura 1: Imagens retiradas do banco de dados IIIT $5 \mathrm{~K}$-Word dataset e utilizadas para o experimento do presente artigo

Peak Signal to Noise Ratio (PSNR $\uparrow$ ):

$$
\text { PSNR }=10 \log _{10} \frac{\left(2^{n}-1\right)^{2}}{\sqrt{M S E}}
$$

Structural Content $(\mathrm{SC} \downarrow)$ :

$$
S C=\frac{\sum_{i=1}^{I} \sum_{j=1}^{J}(P(i, j))^{2}}{\sum_{i=1}^{I} \sum_{j=1}^{J}(\widehat{P}(i, j))^{2}}
$$

[Etapa 4] Análise Estatística:

Para análise dos resultados são utilizadas técnicas estatísticas. São aplicados testes de validação de hipóteses, onde o principal 
objetivo da análise estatística é verificar se existe significância estatística entre os tratamentos aplicados nos dados (Bussab and Morettin; 2010). Para isso são apresentadas duas hipóteses:

Hipótese Nula: $H_{0}$, se esta hipótese é rejeitada, aceita-se a hipótese alternativa;

Hipótese Alternativa: $H_{1}$.

Os testes de hipóteses adotados foram não paramétricos de Friedman (Hodges and Lehmann; 1962) e Wilcoxon (Wilcoxon; 1945), visto que não há garantia de normalidade e homocedasticidade dos dados. $\mathrm{O}$ teste de Friedman não paramétrico é aplicado para apontar diferenças estatísticas entre um grupo de resultados, isto é, entre as funções de agregação utilizadas.

Seja $\mu$ a mediana da medida de qualidade de cada agregador. O teste de hipótese de Friedman é formulado da seguinte forma:

- Hipótese Nula: $\mu_{1}=\mu_{2}=\ldots=\mu_{6}$

- Hipótese Alternativa: Há diferença entre alguma das medianas comparadas.

Confirmada a existência de diferenças entre grupos, isto é, rejeitada a hipótese nula aceita-se a hipótese alternativa, fazendose necessário um teste post hoc para verificar entre quais grupos existem essas diferenças. Para isso foi utilizado o teste pareado não paramétrico de Wilcoxon que se assemelha ao teste de Friedman. O teste de Wilcoxon realiza uma comparação par a par, onde verifica se a hipótese nula de que os agregadores sejam iguais de forma que se a hipótese nula for rejeitada tem-se que o par comparado apresenta diferenças significativas.

Para realização dos testes de hipóteses definiu-se o nível de significância de $5 \%$, isto é, $\alpha=0.05$ significa que tem-se no máximo $5 \%$ de chances de rejeitar a hipótese nula caso seja verdadeira. A medida adotada para se aceitar ou rejeitar a hipótese é o $p$-valor, onde se $p-$ valor $<\alpha$ rejeita-se a hipótese nula, caso $p-v a l o r>\alpha$ aceita-se a hipótese nula.

A Figura 2 representa o processo de entrada da imagem original até sua saída modificada, passando por uma redução através de uma função de agregação (neste caso integral de Choquet Clássica Eq.(1) com medida fuzzy da potência Eq.(5) considerando $q=$ 0.5). É ilustrado na Figura 2 a entrada de uma imagem $4 \times 4$, considerando janelas de $2 \times 2$, isto é, 4 pixels por janela com stride 2.

Nestas condições, aplica-se a integral de Choquet em cada uma das quatro janelas da imagem, gerando uma imagem reduzida em relação a de entrada, onde agora cada uma das quatro janelas apresenta um pixel. Desta forma, a imagem resultante da agregação torna-se uma imagem $2 \times 2$, conforme ilustrado. Na sequência emprega-se o resize a fim de retornar a mesma dimensão da imagem de entrada.

\section{Resultados e Discussão}

A seguir são apresentados os resultados de cada medida de qualidade de imagem. As tabelas que contém os resultados das médias apresentam em cor azul o nome das funções baseadas em integral de Choquet, por outro lado em cor vermelha as funções usuais na literatura e em negrito apresentam-se os melhores agregadores de cada tipo.

A Tabela 1 mostra um recorte da tabela de dados da medida AD, a fim de ilustrar como os dados são organizados para realização das análises. Para avaliar e comparar as funções de agregação (máximo, média, integral de Choquet clássica e integral de Choquet baseada em Cópula) foi aplicado o teste de Friedman. O qual resultou na rejeição da hipótese nula para todas medidas de qualidade $\mathrm{AD}$, $\mathrm{SC} \downarrow, \mathrm{NK} \uparrow, \mathrm{MD} \downarrow, \mathrm{NAE} \downarrow, \mathrm{MSE} \downarrow$ e PSNR $\uparrow$, implicando portanto,
Entrada: Imagem Original

\begin{tabular}{|c|c|c|c|}
\hline 66 & 32 & 76 & 82 \\
\hline 20 & 0 & 15 & 53 \\
\hline 15 & 241 & 230 & 147 \\
\hline 8 & 13 & 255 & 196 \\
\hline \multicolumn{4}{|c}{ Agregação } \\
\hline
\end{tabular}

Imagem Reduzida pela Agregação
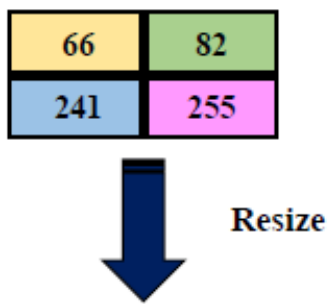

Saída: Imagem Redimensionada

\begin{tabular}{|c|c|c|c|}
\hline 66 & 66 & 82 & 82 \\
\hline 66 & 66 & 82 & 82 \\
\hline 241 & 241 & 255 & 255 \\
\hline 241 & 241 & 255 & 255 \\
\hline
\end{tabular}

Figura 2: Etapas do Processo: entrada e saída de uma imagem utilizando a função máxima. Fonte: Autores.

na existência de diferenças estatísticas entre os agregadores em todas as medidas de qualidade.

Foi possível observar que as funções de agregação máximo e integral de Choquet clássica obtiveram melhores médias para as medidas de qualidade AD Tabela (2), NK $\uparrow$ Tabela (6), SC $\downarrow$ Tabela (4) e MD $\downarrow$ Tabela (3). Como a hipótese nula do teste de Friedman foi rejeitada para as medidas de qualidade, isto é, há diferenças estatísticas entre as funções de agregação aplicadas em cada medida de qualidade.

$\mathrm{O}$ teste post-hoc aplicado em cada uma das medidas $\mathrm{AD}, \mathrm{NK} \uparrow$, $\mathrm{SC} \downarrow$ e $\mathrm{MD} \downarrow$ indicou que apenas para $\mathrm{NK} \uparrow$ a função máximo e integral de Choquet não apresentam diferenças significativas. Com isso, pode-se concluir que, estatisticamente, as duas funções que obtiveram os melhores resultados para as medidas de qualidade $\mathrm{AD}, \mathrm{NK} \uparrow, \mathrm{SC} \downarrow$ e $\mathrm{MD} \downarrow$ são diferentes, e apresentaram resultados superiores em relação às funções média e integral de Choquet baseada em Cópula.

Por outro lado, para as medidas de qualidade PSNR $\uparrow$ Tabela (8), NAE $\downarrow$ Tabela (5) e MSE $\downarrow$ Tabela (7) as médias de cada função de agregação apontaram os melhores resultados para a função média e integral de Choquet baseada em Cópula. Aplicando-se o teste de Friedman, a hipótese nula foi rejeitada para as medidas PSNR $\uparrow$, NAE $\downarrow$ e MSE $\downarrow$. Ou seja, também há diferenças estatísticas entre as funções de agregação para cada uma dessas medidas. O teste pareado de Wilcoxon aplicado em cada uma das medidas PSNR $\uparrow$, $\mathrm{NAE} \downarrow$ e MSE $\downarrow$ indicam que existem diferenças estatísticas entre todos os agregadores para as medidas de qualidade PSNR $\uparrow$, NAE $\downarrow$ e MSE $\downarrow$.

Algo interessante que pode ser observado é que para $\mathrm{AD}, \mathrm{NK} \uparrow$, 
Tabela 1: Recorte da Tabela de Dados da Medida de Qualidade AD (resultado deve ser igual ou próximo de zero). Fonte: Autores

\begin{tabular}{c|c|c|c|c|c|c|c}
\hline Imagem & Stride & $\mathrm{q}$ & $\mathrm{W}$ & $\mathfrak{C}_{\mathfrak{m}}$ & $\mathfrak{C}_{\mathfrak{m}}^{T_{M}}$ & Max & Mean \\
\hline $127 \_1$ & 2 & 0.1 & 2 & -3.5849 & -6.8215 & -8.6169 & -0.1210 \\
$127 \_2$ & 2 & 0.1 & 2 & -4.2975 & -5.7222 & -11.4236 & -0.1309 \\
$127 \_3$ & 2 & 0.1 & 2 & -13.758 & -21.0464 & -6.4345 & -0.1191 \\
$138 \_4$ & 2 & 0.1 & 2 & -32.0959 & -41.1846 & -14.263 & -0.1245 \\
$138 \_5$ & 2 & 0.1 & 2 & -30.451 & -38.3909 & -16.3074 & -0.1319 \\
$138 \_6$ & 2 & 0.1 & 2 & -26.337 & -36.7698 & -15.1768 & -0.1313 \\
$159 \_1$ & 2 & 0.1 & 2 & 3.0724 & 22.473 & -20.9338 & -1.0202 \\
$161 \_1$ & 2 & 0.1 & 2 & -15.1256 & -9.4365 & -15.9019 & -0.1277 \\
$161 \_2$ & 2 & 0.1 & 2 & -13.2427 & -5.0373 & -15.4228 & -0.1258 \\
$52 \_1$ & 2 & 0.1 & 2 & -15.7669 & 4.5155 & -30.7347 & -0.4167 \\
52_2 & 2 & 0.1 & 2 & -27.9197 & -10.2939 & -33.6188 & -0.6948 \\
$52 \_3$ & 2 & 0.1 & 2 & -14.6652 & -4.7945 & -18.5452 & -1.1326 \\
\hline
\end{tabular}

Tabela 2: Médias da Medidas de Qualidade de Imagem AD para cada agregador. Fonte: Autores

\begin{tabular}{c|c|c|c}
\hline \multicolumn{4}{c}{ AD (deve ser próximo de zero) } \\
\hline $\mathfrak{C}_{\mathfrak{m}}$ & $\mathfrak{C}_{\mathfrak{m}}^{T_{M}}$ & Max & Mean \\
\hline-23.0636 & $\mathbf{- 1 8 . 5 3 8 7}$ & -28.1740 & $\mathbf{1 . 4 9 3 0}$ \\
\hline
\end{tabular}

Tabela 3: Médias da Medidas de Qualidade de Imagem MD $\downarrow$ para cada agregador. Fonte: Autores

\begin{tabular}{c|c|c|c}
\hline \multicolumn{4}{c}{$\mathrm{MD} \downarrow$} \\
\hline $\mathfrak{C}_{\mathfrak{m}}^{\mathfrak{c}}$ & $\mathfrak{C}_{\mathfrak{m}}^{T_{M}}$ & Max & Mean \\
\hline $\mathbf{1 6 4 . 9 4 4 4}$ & 188.4722 & $\mathbf{1 3 4 . 3 0 5 6}$ & 182.6667 \\
\hline
\end{tabular}

Tabela 4: Médias da Medidas de Qualidade de Imagem SC $\downarrow$ para cada agregador. Fonte: Autores

\begin{tabular}{c|c|c|c}
\hline \multicolumn{4}{|c}{$\mathrm{SC} \downarrow$} \\
\hline $\mathfrak{C}_{\mathfrak{m}}$ & $\mathfrak{C}_{\mathfrak{m}}^{\mathfrak{T}_{M}}$ & Max & Mean \\
\hline $\mathbf{0 . 7 4 7 7}$ & 0.8267 & $\mathbf{0 . 7 1 8 1}$ & 1.0961 \\
\hline
\end{tabular}

Tabela 5: Médias da Medidas de Qualidade de Imagem NAE $\downarrow$ para cada agregador. Fonte: Autores

\begin{tabular}{c|c|c|c}
\hline \multicolumn{4}{|c}{$\mathrm{NAE} \downarrow$} \\
\hline $\mathfrak{C}_{\mathfrak{m}}$ & $\mathfrak{C}_{\mathfrak{m}}^{T_{M}}$ & Max & Mean \\
\hline 0.3436 & $\mathbf{0 . 3 3 7 2}$ & 0.3489 & $\mathbf{0 . 1 9 9 0}$ \\
\hline
\end{tabular}

Tabela 6: Médias da Medidas de Qualidade de Imagem NK $\uparrow$ para cada agregador. Fonte: Autores

\begin{tabular}{c|c|c|c}
\hline \multicolumn{4}{|c}{$\mathrm{NK} \uparrow$} \\
\hline $\mathfrak{C}_{\mathfrak{m}}$ & $\mathfrak{C}_{\mathfrak{m}}^{T_{M}}$ & Max & Mean \\
\hline $\mathbf{1 . 1 0 3 6}$ & 1.0627 & $\mathbf{1 . 1 1 6 7}$ & 0.9127 \\
\hline
\end{tabular}

$\mathrm{SC} \downarrow$ e MD $\downarrow$ as duas melhores funções foram o máximo e a integral de Choquet Clássica. Por outro lado, para PSNR $\uparrow, N A E \downarrow$ e MSE $\downarrow$ as duas melhores funções foram a média e a integral de Choquet
Tabela 7: Médias da Medidas de Qualidade de Imagem MSE $\downarrow$ para cada agregador. Fonte: Autores

\begin{tabular}{c|c|c|c}
\hline \multicolumn{4}{c}{ MSE $\downarrow$} \\
\hline $\mathfrak{C}_{\mathfrak{m}}$ & $\mathfrak{C}_{\mathfrak{m}}^{T_{M}}$ & Max & Mean \\
\hline 3069.48 & $\mathbf{2 5 8 1 . 1 0}$ & 4103.56 & $\mathbf{1 4 9 3 . 5 9}$ \\
\hline
\end{tabular}

Tabela 8: Médias da Medidas de Qualidade de Imagem PSNR $\downarrow$ para cada agregador. Fonte: Autores

\begin{tabular}{c|c|c|c}
\hline \multicolumn{4}{c}{ PSNR $\downarrow$} \\
\hline $\mathfrak{C}_{\mathfrak{m}}$ & $\mathfrak{C}_{\mathfrak{m}}^{T_{M}}$ & Max & Mean \\
\hline 14.1419 & $\mathbf{1 4 . 5 3 4 4}$ & 13.2301 & $\mathbf{1 7 . 2 1 8 7}$ \\
\hline
\end{tabular}

baseada em Cópula.

As Figuras 3 e 4 mostram a imagem de entrada (original) e a saída de cada uma das funções de agregação considerando-se: janela $2 \times 2$, stride 2 e $q=0.5$. Os valores das imagens da função máximo e integral de Choquet na maioria dos casos estiveram mais próximos em comparação com os resultados das funções média aritmética e Cópula, como visto nos testes. Porém, é possível verificar diferenças visuais na integridade das imagens resultantes em comparação à original.

Tanto na Figura 3 quanto na Figura 4 observa-se, por exemplo, que a imagem do máximo apesar de apresentar bons resultados nas medidas de qualidade visivelmente não é tão próxima a original, pois suas bordas estão mais serrilhadas.

\section{Considerações Finais}

O processo ilustrado na Figura 2 é clássico na literatura mudandose apenas tamanhos de janelas e strides. O diferencial deste trabalho está em apresentar funções de agregação baseadas na integral de Choquet como alternativa as funções clássicas como máximo e média. Os resultados da aplicação da integral de Choquet em redimensionalização mostram-se promissores. Foi possível observar que a integral de Choquet Clássica se assemelha ao máximo, e a integral de Choquet baseada em Cópula com a média. Verificou-se, ainda, que a integral de Choquet resultou em imagens de qualidade e manteve a integridade da imagem original. Isto é, foi capaz de minimizar problemas de bordas serrilhadas em linhas oblíquas/diagonais, embora elas não sejam as mais similares com a imagem original conforme mostrado com as medidas de 


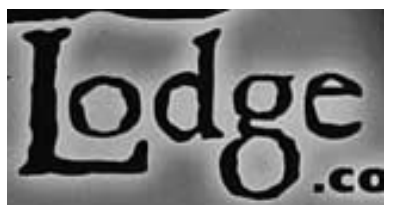

Entrada

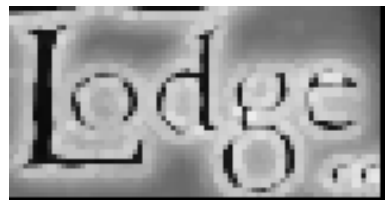

Maximo

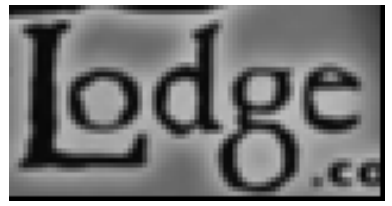

Media

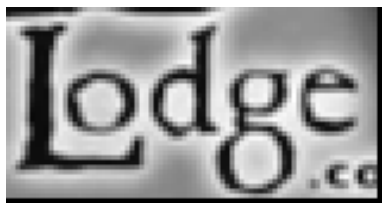

integraldeChoquet

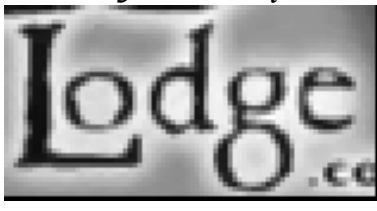

Copula

Figura 3: Resultados de máximo, média, integral de Choquet e cópula obtidos através dos experimentos aplicados a uma das imagens do conjunto de dados IIIT 5K-Word chamado 138_6 (após o processo de redimensionamento). Os parâmetros utilizados foram: tamanho da janela $=\overline{4} \times 4$, stride $=2$ e expoente da medida fuzzy $=0.7$

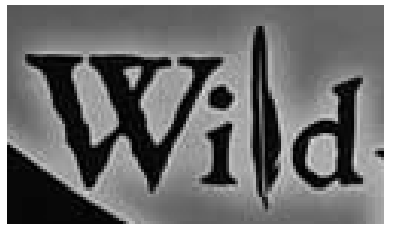

Entrada

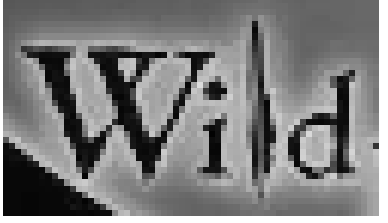

Maximo

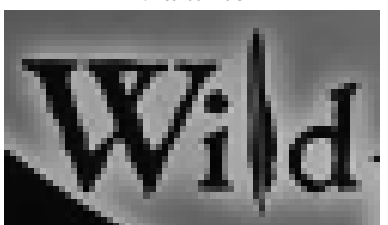

Media

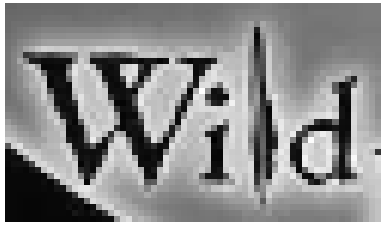

Choquetintegral

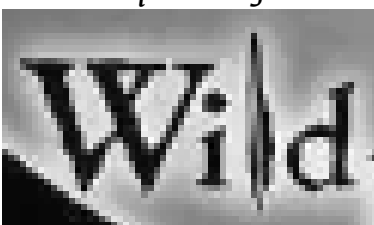

Copula

Figura 4: Resultados de maximo, media, integral de Choquet e pula obtidos através das experiências aplicadas a uma das imagens do conjunto de dados IIIT 5K-Word chamado 138_4 (após o processo de redimensionamento). Os parâmetros utilizados foram: tamanho da janela $=\overline{2} \times 2$, stride $=2$ e expoente da medida fuzzy $=0.1$

qualidade.

Em geral, os resultados apresentados no trabalho mostramse prósperos tendendo a refinar os estudos, de modo que o parâmetro $q$ utilizado na integral do Choquet em relação à medida fuzzy da potência pode ser aprendido por algum algoritmo, como algoritmo genético ou até mesmo redes neurais, gerando resultados superiores. Em trabalhos futuros pretende-se utilizar funções de pré-agregação apresentadas nos trabalhos de (Lucca et al.; 2016) e (Lucca et al.; 2018) de forma a refinar a escolha do parâmetro $q$ da medida fuzzy.

\section{Agradecimentos}

As autoras Camila Alves Dias e Jéssica C. Saldivia Bueno agradecem à CAPES pelo apoio financeiro recebido. Graçaliz P. Dimuro tem financiamento do CNPq (processo 305882/2016-3). Eduardo N. Borges tem financiamento da FAPERGS (TO 17/25510000872-3).

\section{Referências}

Alsina, C., Frank, M. J. and Schweizer, B. (2006). Associative Functions: triangular Norms and Copulas, World Scientific.

Beliakov, G., Sola, H. B. and Sanchez, T. C. (2016). A Practical 
Guide to Averaging Functions, Springer.

Bussab, W. O. and Morettin, P. A. (2010). Estatística Básica, São Paulo: Editora Saraiva.

Choquet, G. (1999). Theory of capacities, Annales de l'Institut Fourier (5): 131-295.

Eskicioglu, A. M. and Fisher, P. S. (1995). Image quality measures and their performance, IEEE Transactions on communications 43(12): 2959-2965.

Grabisch, M., Marichal, J., Mesiar, R. and Pap, E. (2009). Aggregation Functions, Cambridge University Press, Cambridge.

Hodges, J. L. and Lehmann, E. L. (1962). Rank methods for combination of independent experiments in analysis of variance, The Annals of Mathematical Statistics 33(2): 482-497.

Klement, E. P., Mesiar, R. and Pap, E. (2000). Triangular Norms, Kluwer Academic Publisher, Dordrecht.

Lucca, G., Sanz, J. A., Dimuro, G. P., Bedregal, B., Asiain, M. J., Elkano, M. and Bustince, H. (2017). Cc-integrals: Choquet-like copula-based aggregation functions and its application in fuzzy rule-based classification systems, Knowledge-Based Systems (119): 32-43.

Lucca, G., Sanz, J. A., Dimuro, G. P., Bedregal, B., Mesiar, R. and Bustince, A. K.-R. H. (2016). Preaggregation functions: Construction and an application., IEEE Transactions on Fuzzy Systems 24(2): 260-272.

Lucca, G., Sanz, J. A., Dimuro, G. P., BedregalL, B., Bustince, H. and Mesiar, R. (2018). Cf-integrals: A new family of pre-aggregation functions with application to fuzzyrule-based classification systems., Information Sciences (435): 94-110.

Scherer, D., Muller, A. and Behnke, S. (2010). Evaluation of pooling operations in convolutional architectures for object recognition, International Conference on Artificial Neural Networks pp. 92-101.

Wilcoxon, F. (1945). Individual comparisons by ranking methods, Biometrics bulletin 1(6): 80-83. 